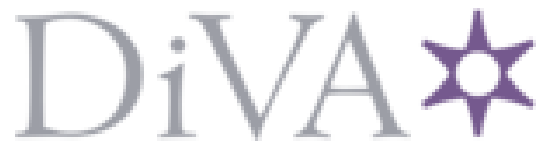

http://www.diva-portal.org

This is the published version of a paper published in British Journal of Dermatology.

Citation for the original published paper (version of record):

Alexeyev, O A. (2019)

Psoriasis, gut and microbiome

British Journal of Dermatology, 181(6): 1126-1126

https://doi.org/10.1111/bjd.18467

Access to the published version may require subscription.

N.B. When citing this work, cite the original published paper.

Permanent link to this version:

http://urn.kb.se/resolve?urn=urn:nbn:se:umu:diva-166806 
4 Nakamura T, Haider S, Colicino S et al. Different definitions of atopic dermatitis: impact on prevalence estimates and associated risk factors. Br J Dermatol 2019; 181:1272-79.

5 Andersen YMF, Egeberg A, Hamann CR et al. Poor agreement in questionnaire-based diagnostic criteria for adult atopic dermatitis is a challenge when examining cardiovascular comorbidity. Allergy 2018; 73:923-31.

\section{Psoriasis, gut and microbiome}

DOI: $10.1111 /$ bjd. 18467

Linked Article: Hidalgo-Cantabrana et al. Br J Dermatol 2019; 181:1287-1295.

'All disease begins in the gut', the phrase attributed to Hippocrates, may sound like an overstatement but empirical evidence has linked many chronic metabolic and immune disorders to gut malfunction. It is increasingly recognized that the microbiome can change or switch on a wide range of diseases including cancer, cardiometabolic diseases, allergies, skin diseases and obesity. ${ }^{1}$ This is not surprising because recent advances in microbiome research have revealed that microbial cells outnumber human cells in our body by a ratio of around $10: 1$.

In this issue of the BJD, Hidalgo-Cantabrana et al. report on lower bacterial diversity and different relative abundance of bacterial taxa in patients with psoriasis. ${ }^{2}$ They further suggest that gut microbiota dysbiosis could be one of the triggers in the manifestation of psoriasis in genetically predisposed individuals. The microbiome of patients with psoriasis has been explored and reported in earlier studies. The advantage of this study is the inclusion of a healthy cohort from the same geographical area to minimize the environmental impact. While the presence of gut dysbiosis appears to be common in most studies of psoriasis, the relative abundance/decrease of different genera varies, most likely due to sequencing methodologies and computational analysis.

Essentially, the 16S rRNA gene sequencing of gut microbiome represents only the first step of a scientific journey to reveal what kind of bacteria are in our gut; it provides a solid foundation for further scientific exploration and knowledge building. However, the clinical significance of organisms identified solely by $16 \mathrm{~S}$ rDNA gene sequencing is unclear. In addition to viable cells, dormant and dead bacteria, as well as extracellular DNA, are targeted. The next essential step is to map the presence and quantity of live bacteria. This analysis may not necessarily yield the same results as 16S rRNA gene sequencing. Another limiting factor in this study is enrolling a patient cohort with established disease and a long clinical history (average of 25 years), making it difficult to establish any meaningful cause-effect relationship.

The ultimate goal of any clinical research is the translation of clinical discoveries into new diagnostics, treatment and preventive approaches. The gut microbiome is an attractive therapeutic target because, theoretically, there is good amenability of the gut microbiome. Therapies could be based on environmental changes, diets, drugs or probiotic supplements. Evidence suggests that changing a diet dramatically leads to relatively quick gut microbiome changes, in which around $30-40 \%$ of the bacterial strains vary in their abundance. ${ }^{3}$ The big vision is to modulate or even prevent diseases via the microbiome alone or with other life factors. In this way, microbiome research is moving from an isolated offside position to become a beacon of hope with a lot of potential and possibilities.

Department of Pathology, Umeå University,

O. Alexeyev

90185 Umea, Sweden

E-mail: oleg.alexeyev@umu.se

\section{References}

1 Honda K, Littman DR. The microbiota in adaptive immune homeostasis and disease. Nature 2016; 535:75-84.

2 Hidalgo-Cantabrana C, Gómez J, Delgado S et al. Gut microbiota dysbiosis in a cohort of patients with psoriasis. Br J Dermatol 2019; 181:1287-95.

3 Singh RK, Chang HW, Yan D et al. Influence of diet on the gut microbiome and implications for human health. J Transl Med 2017; 15:73.

Conflicts of interest: none to declare.

\section{$\mathrm{CD}^{+}$mycosis fungoides: a wolf in sheep's clothing?}

DOI: $10.1111 /$ bjd. 17881

Linked Article: Doerschner et al. Br J Dermatol 2019;
181:1296-1302.

In this issue of the BJD, Doerschner and colleagues report on a case of mycosis fungoides (MF) with involvement of the central nervous system (CNS). Complete and ongoing remission of CNS lesions for 17 months was achieved with systemic chemotherapy and autologous stem-cell transplantation (SCT). Interferon (IFN) alfa-2a was used successfully to treat the subsequent relapse confined to the skin. ${ }^{1}$

Initially, the patient was timely diagnosed with advancedstage MF of 5 months' duration restricted to the skin. Of note, the Prospective Cutaneous Lymphoma International Prognostic Index (PROCLIPI) study has demonstrated a median diagnostic delay of 36 months in early-stage MF between first symptoms and initial diagnosis. ${ }^{2}$ However, in the face of currently lacking biomarkers for predictable disease progression it is debatable whether patients will benefit from early diagnosis. Moreover, accurate patient education on their prognosis is challenging with 5 -year survival rates ranging from $88 \%$ to $30 \%$ dependent 